weave empirical observations into a coherent picture. He also used state-of-the-art technology to build and develop instruments of scientific observation, such as the telescope and the microscope, that, through their widespread use, went on to fundamentally influence later science.

The book is remarkable for its clarity, precision and historical accuracy. Numerous drawings, figures and photographs help the reader pick a path through the historical and scientific reconstruction.

Nowadays scientific knowledge is extraordinarily developed and university education promotes hyper-specialization. Scientists who end up working in two different fields may struggle, owing to an insufficient depth of knowledge in either one. This book reminds us that things have not been always this way: as the authors note in their introduction, the excerpts from
Galileo's writings have been chosen to offer a vision of his multiple interests in many scientific and cultural fields, "of his prodigious curiosity for all natural phenomena, of his inexhaustible capacity for posing questions to himself and searching for answers by reasoning. And also of his exultation at making discoveries."

Giorgio Parisi is in the Department of Physics, University 'La Sapienza', Piazzale Aldo Moro 2, Rome 00185, Italy.

\title{
A drink from the magic well
}

\section{Asian Honey Bees: Biology, Conservation and Human Interactions \\ by Benjamin P. Oldroyd \& Siriwat Wongsiri Harvard University Press: 2006. 340 pp. $\$ 59.95, € 38.95$}

\section{Francis L. W. Ratnieks}

Karl von Frisch, who won the 1973 Nobel Prize in Physiology or Medicine for discovering the honeybee dance language, called the honeybee a magic well - no matter how much was discovered, more remained. Most research on honeybees, including von Frisch's, has been done on Apis mellifera. This species, the western hive bee, is native to Europe, Africa and the Middle East, and has been introduced worldwide because of its economic importance in making honey and pollinating crops. But Asia is home to eight more species of Apis, five of which have been recently discovered or recognized. The past ten years has seen an upsurge of research on these species, leading to some 250 scientific articles.

Four of the Asian honeybee species nest, like $A$. mellifera, in cavities within which they build multiple vertical wax combs. There are also two species each of dwarf and giant honeybees. These build a single vertical wax comb, usually under a branch or, in the case of giant bees, an overhang. All live in tropical Asia, except for the eastern hive bee, A. cerana, which extends as far north as Japan and Korea, and the giant mountain honeybee, A. laboriosa, which lives in the Himalayas.

Asian Honey Bees has a simple but effective structure. It begins with chapters on honeybees in general, the Asian species, honeybee evolution, speciation and biogeography. These provide the context in which to understand the biodiversity and evolution of honeybees and also for the following five chapters on particular topics: dance communication and foraging; reproduction; worker sterility and kin selection; nesting and defence; and parasites and predators. The final two chapters address interactions with humans and conservation.

Studies of Asian honeybees have done much to elucidate the evolution of the dance language, a research question initiated more than 50 years ago by von Frisch's student Martin Lindauer, and many other areas of honeybee

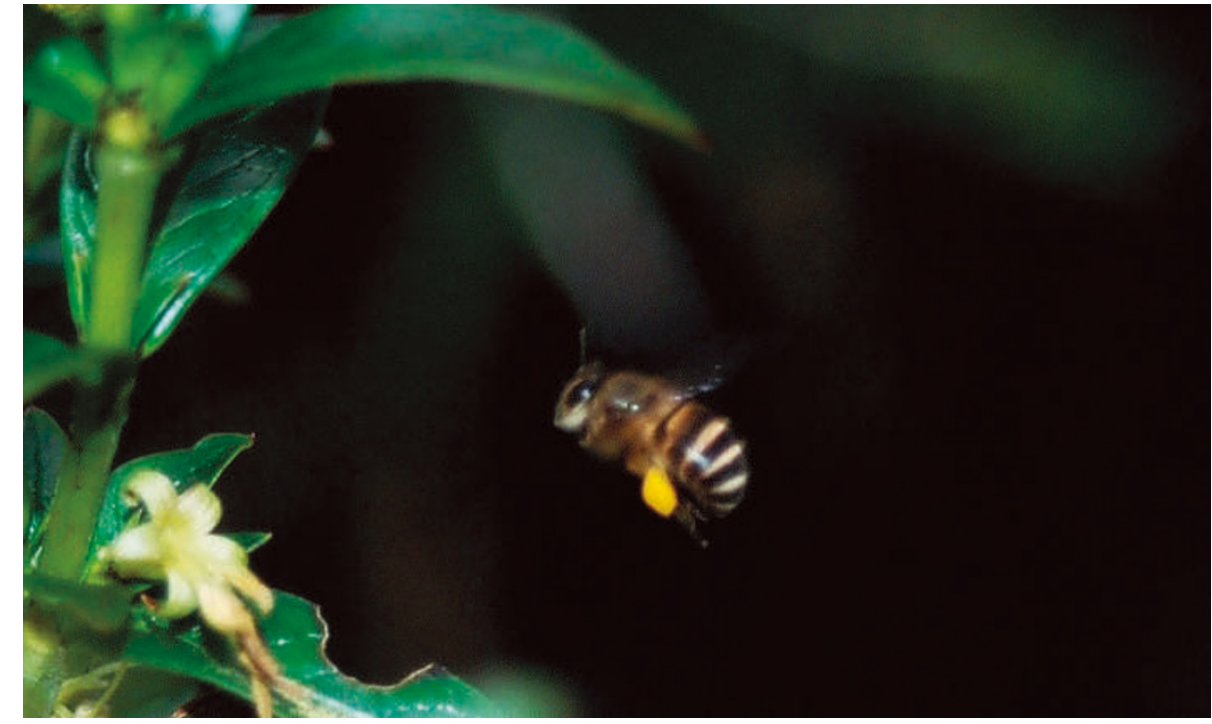

Never mind the buzz about Apis mellifera - A. nuluensis is one of eight other honeybee species in Asia.

biology. These studies have also discovered examples of nature at its most wonderful, of which two deserve a mention here. Workers of $A$. cerana defend their nest against giant hornets by encasing the hornet in a living ball of bees, which generate heat and literally cook the hornet to death before it can send a pheromone signal to recruit more hornets to the attack. Indeed, the scout hornet's pheromone alerts the bees. Colonies of giant honeybees, A. dorsata, often migrate $100 \mathrm{~km}$. At certain times of the year the bees abandon their nesting trees, flying to better areas for a season before returning. Genetic studies show that the same colony with the same queen may return months later to the same tree.

Asian Honey Bees succeeds admirably. It is both an authoritative monograph that will satisfy experts and a highly readable book that will engage students and biologists in general. It presents Asian honeybees in the broader context of social behaviour and evolution while giving a real feeling for the bees themselves, including their interactions with humans you can buy bee brood in many Asian food markets, something the authors have used as a way of getting samples.

The book itself is well produced, although I have one quibble. The numerous photos are reproduced in black and white. Surely insects demand colour! But this is partly offset by a stunning cover photograph of a nest of the giant honeybee, $A$. dorsata.

Francis Ratnieks is in the Laboratory of Apiculture and Social Insects, Department of Animal and Plant Sciences, University of Sheffield,

Sheffield S10 2TN, UK

\section{MORE ON BEES}

Letters from the Hive: An Intimate History of Bees, Honey, and Humankind

by Stephen Buchmann with Banning Repplier (Bantam Books, \$14)

A book interweaving the natural history of bees with their small but vital place in world culture. The story ranges from prehistoric honey-hunting to mead-making, pollination, bees in warfare, and the medicinal properties of honey.

\section{A Keeper of Bees: Notes on Hive and Home} by Allison Wallace (Random House, \$23.95)

A beautifully written book that peers into the hive for a hard look at honeybee behaviour, then swerves all over the place, covering the events that first sparked the author's interest in bees, the effects of pollution, bees' place in myth and nature, and the many hives the author has created over the years in various back gardens. 